OPEN ACCESS

Edited by:

Yang Yang,

Northwest University, China

Reviewed by:

Hermona Soreq,

Hebrew University of Jerusalem, Israel Xiaoqiang Tang,

Sichuan University, China

${ }^{*}$ Correspondence:

Yun-Cheng Wu

yunchw@medmail.com.cn;

drwu2006@163.com

Specialty section:

This article was submitted to

Neuroendocrine Science,

a section of the journal

Frontiers in Neuroscience

Received: 31 July 2018

Accepted: 24 October 2018

Published: 21 November 2018

Citation:

Zhang J-F, Zhang $Y-L$ and Wu Y-C (2018) The Role of Sirt1 in Ischemic

Stroke: Pathogenesis and Therapeutic

Strategies. Front. Neurosci. 12:833.

doi: 10.3389/fnins.2018.00833

\section{The Role of Sirt1 in Ischemic Stroke: Pathogenesis and Therapeutic Strategies}

\author{
Jun-Fang Zhang, Yu-Lei Zhang and Yun-Cheng $W u^{*}$ \\ Department of Neurology, Shanghai General Hospital, Shanghai Jiao Tong University School of Medicine, Shanghai, China
}

Silent mating type information regulation 2 homolog 1 (Sirt1), a nicotine adenine dinucleotide $\left(\mathrm{NAD}^{+}\right)$-dependent enzyme, is well-known in playing a part in longevity. Ischemic stroke is a major neurological disorder and is a leading cause of death and adult disability worldwide. Recently, many studies have focused on the role of Sirt1 in ischemic stroke. Numerous studies consider Sirt1 as a protective factor and investigate the signaling pathways involved in the process under ischemic stress. However, the answer to whether upregulation of Sirt1 improves the outcome of stroke is still a controversy. In this review, we discuss the role and mechanisms of Sirt1 in the setting of ischemic stroke.

Keywords: Sirt1, deacetylase, ischemic stroke, neuroprotection, sirtuin

\section{INTRODUCTION}

Stroke is the second leading cause of death and a major cause of adult disability worldwide (Cushman et al., 2008; Donnan et al., 2008). Ischemic stroke is the most common type of stroke and accounts for $87 \%$ of all stroke cases (Macrez et al., 2011). Vascular recanalization therapies, including tissue plasminogen activator thrombolysis and thromboembolectomy, are currently considered the best therapies but are limited due to a narrow treatment window and several safety concerns (Del Zoppo et al., 2009; Albers et al., 2018; Nogueira et al., 2018; Thomalla et al., 2018). There is no effective treatment for ischemic stroke so far. It is crucial to find new therapies for this major medical problem.

There are two major injuries to the brain. Firstly, a blocked cerebral artery and secondly, the subsequent reperfusion may cause the secondary injury. Penumbra, defined as a zone of tissue surrounding the core of the infarction area, is an important target for researchers and clinicians to find effective therapies (Wang et al., 2012). The occlusion of a cerebral artery can cause the deprivation of oxygen and energy, thereby leading to the dysfunction of cerebral tissue and neuron death. After the early phase of necrosis, the following pathophysiological reactions such as formation of free radicals, changes in gene expression, apoptosis, and inflammation contribute to the delayed phase of tissue damage (Moskowitz et al., 2010; Petegnief and Planas, 2013). However, the ineffectiveness of current therapies indicates that there should be other important mechanisms leading to the pathophysiology of ischemic stroke.

Silent mating type information regulation 2 homolog 1 (Sirt1), also called sirtuin 1 , is a nicotine adenine dinucleotide $\left(\mathrm{NAD}^{+}\right)$-dependent enzyme (Kelly, 2010). Among several potential 
TABLE 1 | Role and mechanisms of Sirt1 in ischemic stroke.

\begin{tabular}{|c|c|c|c|}
\hline Compound & SIRT1 role & Mechanism & Reference \\
\hline IRF9 & Anti-apoptosis & $\begin{array}{l}\text { IRF9 inhibits Sirt1 deacetylase activity, culminating in the } \\
\text { acetylation and activation of p53-mediated cell death } \\
\text { signaling in response to acute I/R stress. }\end{array}$ & Zhang et al., 2017 \\
\hline LKE & Anti-apoptosis, anti-inflammation & $\begin{array}{l}\text { LKE mediated, at least in part, through CRMP2 and Sirt1 } \\
\text { upregulation and PARP1 inhibition. }\end{array}$ & Nada et al., 2012 \\
\hline \multirow[t]{3}{*}{ Resveratrol } & Anti-oxidation, anti-apoptosis, anti-inflammation & $\begin{array}{l}\text { Resveratrol upregulates the Sirt1/PGC-1a, Akt/pCREB, and } \\
\text { p38 pathways and downregulates pERK1/2 expression in } \\
\text { ischemic injury. }\end{array}$ & Zhu et al., 2010 \\
\hline & Regulation in glycolytic function & $\begin{array}{l}\text { Resveratrol via neuronal Sirt1 promotes glycolytic efficiency } \\
\text { to combat energetic stress. }\end{array}$ & Yue et al., 2008 \\
\hline & Energy regulation & $\begin{array}{l}\text { Resveratrol provides neuroprotection by inhibiting PDEs } \\
\text { and regulating the CAMP/AMPK/Sirt1 pathway. }\end{array}$ & $\begin{array}{l}\text { Kundu and } \\
\text { Thompson, } 2008\end{array}$ \\
\hline Resveratrol preconditioning & Anti-oxidation and regulation of neural survival & $\begin{array}{l}\text { The mechanism is mediated by Sirt1 through upregulation } \\
\text { of BDNF and downregulation of uncoupling protein } 2 \text {. }\end{array}$ & $\begin{array}{l}\text { Koronowski et al., } \\
2015\end{array}$ \\
\hline HBO-PC & Anti-oxidation & $\begin{array}{l}\text { HBO-PC is mediated by the activation of Sirt1 and } \\
\text { Nrf2/antioxidant defense pathway. }\end{array}$ & Xue et al., 2016 \\
\hline Arctigenin & Anti-inflammation & $\begin{array}{l}\text { Arctigenin inhibited NLRP3 inflammasome activation } \\
\text { through Sirt1 pathway. }\end{array}$ & $\begin{array}{l}\text { Hernandez- } \\
\text { Jimenez et al., } \\
2013\end{array}$ \\
\hline Curcumin & Anti-apoptosis & $\begin{array}{l}\text { Curcumin activates Sirt1 signaling, resulting in decreased } \\
\text { expression of Ac-p53 and Bax and increased Bcl-2 } \\
\text { expression. }\end{array}$ & Miao et al., 2016 \\
\hline Icariin & Anti-oxidation & $\begin{array}{l}\text { Icariin protects against brain ischemic injury by increasing } \\
\text { the Sirt1 and PGC-1a expression. }\end{array}$ & Kou et al., 2017 \\
\hline \multirow[t]{2}{*}{ Nampt } & Regulation in autophagy & $\begin{array}{l}\text { Nampt induces autophagy via TSC2-mTOR-S6K1 signaling } \\
\text { pathway in a Sirt1-dependent manner. }\end{array}$ & Wang et al., 2012 \\
\hline & Energy regulation & $\begin{array}{l}\text { Nampt protects against ischemic stroke through rescuing } \\
\text { neurons from death via the SIRT1-dependent AMPK } \\
\text { pathway. }\end{array}$ & $\begin{array}{l}\text { Dasgupta and } \\
\text { Milbrandt, } 2007\end{array}$ \\
\hline Leptin & Anti-apoptosis & $\begin{array}{l}\text { Leptin increases CB2, Sirt1 and TRPV1 expression as well } \\
\text { as expression of the endogenous leptin receptors and } \\
\text { reduces the expression of CB1 receptors. }\end{array}$ & Ashrafi et al., 2017 \\
\hline Magnolol & Anti-apoptosis and anti-inflammation & $\begin{array}{l}\text { Magnolol activation of Sirt1 was accompanied by the } \\
\text { inhibition of Ac-FOXO1 expression, which decreased the } \\
\text { expression of bax and increased Bcl-2 expression. }\end{array}$ & Chen et al., 2014 \\
\hline Melatonin & Anti-apoptosis & $\begin{array}{l}\text { Melatonin increased Sirt1 and reduced Ac-p53 and Ac-NF- } \\
\kappa \mathrm{B} \text { and was also associated with a rise of } \mathrm{Bcl} 2 \text { and a } \\
\text { lowering of Bax. }\end{array}$ & Wang et al., 2008 \\
\hline Tetrahydroxystilbene glucoside & Anti-oxidation and Anti-apoptosis & $\begin{array}{l}\text { The mechanisms are involved with depression of the JNK } \\
\text { and Bcl-2 family-related apoptotic signaling pathway, and } \\
\text { inhibition of iNOS mRNA expression, which was partly } \\
\text { mediated by the activation of Sirt1 and thereby inhibition of } \\
\text { NF-кB activation. }\end{array}$ & Taxin et al., 2014 \\
\hline SalB & Anti-oxidation, anti-apoptosis, anti-inflammation & $\begin{array}{l}\text { SalB decreased TNF- } \alpha \text { and IL-1 levels in the brain tissue } \\
\text { and upregulated the expression of Sirt1 and Bcl-2 and } \\
\text { downregulated the expression of Ac-FOXO1 and Bax. }\end{array}$ & Guo et al., 2017 \\
\hline Estrogen & Energy regulation & $\begin{array}{l}\text { Estrogen protects against ischemic stroke via the } \\
\text { SIRT1-dependent AMPK pathway. }\end{array}$ & Wang et al., 2011 \\
\hline HBO-PC & Anti-apoptosis & $\begin{array}{l}\text { SirT1 increased Bcl-2 expression and decrease cleaved } \\
\text { caspase } 3 .\end{array}$ & Yan et al., 2013 \\
\hline / & Effect on BBB permeability & $\begin{array}{l}\text { Sirt1 inhibited Sirt3 expression through the AMPK-PGC1 } \\
\text { pathway, causing mitochondrial ROS generation and then } \\
\text { increase BBB permeability. }\end{array}$ & $\begin{array}{l}\text { Hurtado et al., } \\
2013\end{array}$ \\
\hline / & Regulation of cerebral blood flow & Sirt1 upregulates the nitric oxide (eNOS-NO) system. & Briski et al., 2014 \\
\hline
\end{tabular}

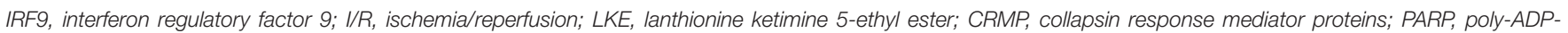

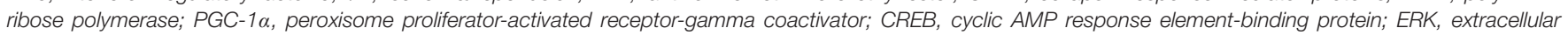

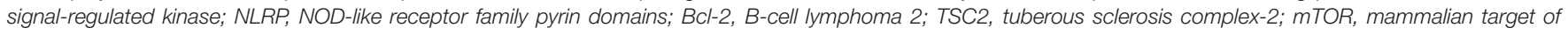

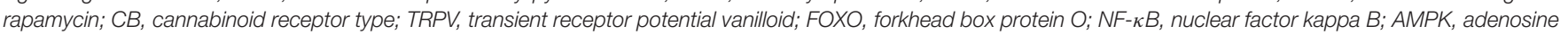

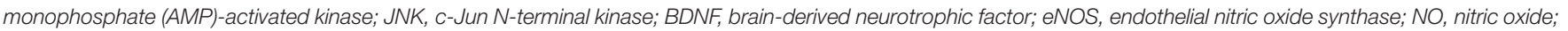
iNOS, inducible NO synthase; BBB, blood-brain barrier; HBO-PC, hyperbaric oxygen preconditioning. 
therapeutic targets, Sirt1 is one of the most valuable candidates because it can modulate gene expression and adapt cell metabolism to ischemic stress. Sirt1 deacetylates numerous transcription factors other than histones and is involved in various biological processes (Chen et al., 2005). A large number of studies consider Sirt1 as a survival factor against aging process, including cardiovascular disease ( $\mathrm{Li}$ et al., 2011) and neurodegeneration (Donmez, 2012). In recent years, Sirtl has been found to be neuroprotective against cerebral ischemia/reperfusion (I/R) injury (Herskovits and Guarente, 2014). Activation of Sirt1 alleviates ischemia through several mechanisms (Table 1). Although it is still a controversy whether Sirtl could improve stroke outcome, there have been plenty of studies indicating the potential therapeutic value of Sirt1 for ischemic stroke. In this review, we discuss the role and potential mechanisms by which Sirtl protects against ischemic stroke (Figure 1).

\section{ANTI-INFLAMMATORY AND ANTI-APOPTOTIC EFFECTS}

Sirt1 plays an important role in endogenous neuroprotection, which is demonstrated using pharmacological and genetic gain of function and loss of function experiments. Activating or inhibiting enzymatic activity of Sirt1 causes a decrease or an increase in infarct volume. Sirtl is considered to be related to anti-inflammatory and anti-apoptotic effects in cerebral ischemia because its inhibition exacerbated ischemic injury accompanied by increased acetylation of p53 and NF- $\mathrm{B}$ (nuclear factor-kappa B p65), which are important factors mediating inflammatory and apoptotic pathways causing brain damage (Hernandez-Jimenez et al., 2013).

Activation of Sirt1 signaling during cerebral ischemia by curcumin (CCM), a compound mainly extracted from Curcuma longa, leads to the decreased expression of Ac-p53 and Bax, increased expression of B-cell lymphoma 2 (Bcl-2), and finally attenuated the inflammation (Miao et al., 2016). Similarly, interventions targeting IRF9 inhibition may help to mediate Sirt1-related ischemic neuron survival through decreased expression of p53 (Chen et al., 2014).

Arctigenin (ARC), a phenylpropanoid dibenzylbutyrolactone lignan derived from Arctium lappa L., was reported to provide neuroprotection against ischemic stroke by inhibiting NLRP3 inflammasome activation through the activation of Sirt1 signaling pathway in the middle cerebral artery occlusion (MCAO) model with decreased infarct volume, neurological scores, and brain water content (Zhang et al., 2017). Another study also used MCAO model to demonstrate that LKE (lanthionine ketimine-5-ethyl ester) protected ischemic brain tissue partly through CRMP2 and Sirt1 upregulation and PARP1 inhibition (Nada et al., 2012).

Hyperbaric oxygen (HBO) therapy is considered to be one of the safe and feasible methods to provide neuroprotective benefits for patients with ischemic stroke. It was demonstrated that Sirt1 was involved in the HBO therapy induced ischemic tolerance and Nrf2 might be the downstream regulator of Sirt1 (Xue et al., 2016). Hyperbaric oxygen-preconditioning was found to upregulate the expression of Sirt1 protein and mRNA after focal cerebral ischemic injury, leading to the suppression of apoptosis. Upregulation of Sirt1 caused an increased expression of antiapopotic Bcl-2 and a decreased pro-apoptotic cleaved caspase-3 in oxygen-glucose deprivation (OGD) injury models (Yan et al., 2013).

\section{ANTIOXIDATION}

Cerebral ischemia was found to produce a large number of free radicals and cause neurotoxicity in I/R injury (Crack and Taylor, 2005). Increased generation of intracellular reactive oxygen species (ROS) such as the hydroxyl radical is demonstrated to induce oxidative stress and mitochondrial enzyme dysfunction, leading to the pathophysiology of damage of cerebral ischemia, and this damage, in turn, aggravates cerebral injury (Mattiasson et al., 2003; Slemmer et al., 2008). Therefore, to prevent intracellular calcium accumulation and further cell apoptosis followed by oxidative stress in ischemic stroke, it is critical to find targets to inhibit such cellular signal transduction pathways (Chan, 2004; Mehta et al., 2007; Doyle et al., 2008; Thompson et al., 2015).

Several studies suggested that Sirt1 plays an important role in oxidative stress in ischemic stroke. Peroxisome proliferatoractivated receptor gamma coactivator 1-alpha $(\mathrm{PGC}-1 \alpha)$ is a potent stimulator of mitochondrial respiration and gene transcription in the liver, heart, skeletal muscle, and neurons (Wareski et al., 2009). Hypoxia increases mRNA levels and the protein expression of PGC-1 $\alpha$ in wild type mice (Gutsaeva et al., 2008). Increased expression of PGC- $1 \alpha$ could reduce neuronal death mediated by oxidative stress (St-Pierre et al., 2006). Sirt1 could directly affect PGC- $1 \alpha$ activity through phosphorylation and deacetylation (Canto and Auwerx, 2009), thereby protecting against ischemia stroke.

Resveratrol (3,5,4'-trihydroxystilbene), a polyphenol found in red wine, can ameliorate neuronal damages caused by cerebral ischemia and neurodegenerative diseases such as Alzheimer's and Parkinson's disease (Sun et al., 2010; Wang et al., 2014; Pasinetti et al., 2015). Resveratrol was demonstrated to protect ischemic stroke by upregulating the Sirt1-PGC-1 $\alpha$ signaling pathways and exert an antioxidative effect under ischemic stress (Shin et al., 2012). Furthermore, resveratrol preconditioning was showed to upregulate BDNF and downregulate uncoupling protein 2 by mediating Sirt1, tolerance in brain (Koronowski et al., 2015). In addition, alpha-lipoic acid (ALA, 1,2-dithiolane3-pentanoic acid), a free radical scavenger in its oxidized state functions as an essential co-factor in the mitochondrial dehydrogenase complexes. Icariin (ICA), one of the major active flavonoids extracted from the Chinese medicinal herb, Epimedium brevicornum Maxim, were also proved to protect against ischemic stroke by increasing Sirt1 and PGC-1 $\alpha$ expression (Zhu et al., 2010; Fu et al., 2014).

Another study found that Sirt1 was upregulated chronically at 14 days after a single resveratrol preconditioning treatment. This phenomenon was associated with negative regulation of 


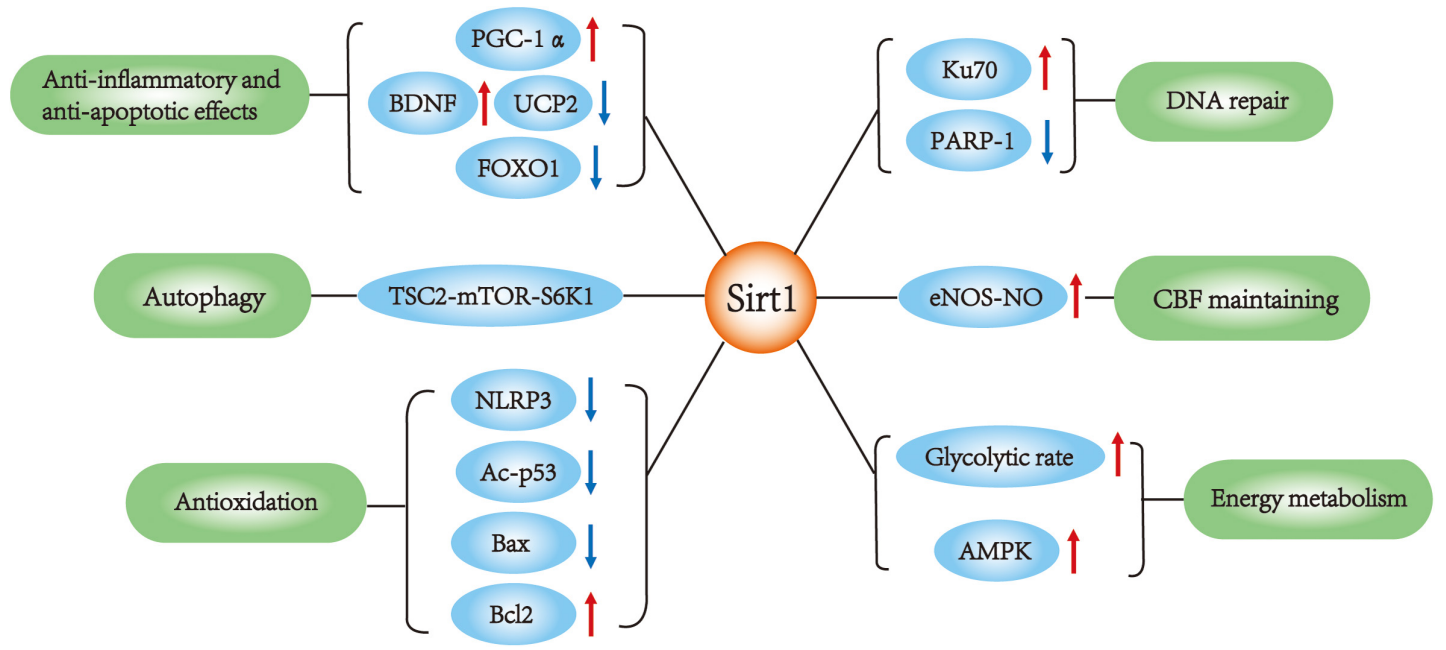

FIGURE 1 | Sirt1 main functions in ischemic stroke. SIRT1 interacts with multiple targets in ischemic stroke, which is involved in the regulation of apoptosis, autophagy, DNA repair, inflammation, metabolism and oxidative stress, cerebral blood flow.

mitochondrial uncoupling protein-2 (UCP2), a proton channel found in the inner mitochondrial membrane that uncouples oxidative phosphorylation (Della-Morte et al., 2009), by binding directly to its promoter (Bordone et al., 2006) and upregulation of brain-derived neurotrophic factor (BDNF), which is an important growth factor that promotes the survival and growth of neurons (Bramham and Messaoudi, 2005).

In addition, salvianolic acid B (Sal B) (Lv et al., 2015), which is the most abundant and bioactive compound of danshen (Salvia miltiorrhiza), and Magnolol (Kou et al., 2017), an organic compound found in the bark of Houpu magnolia (Magnolia officinalis), were demonstrated to activate Sirt1 signaling, accompanied by reduced expression of ac- FoxO1 whose function is to synthesize antioxidants and further help neurons provide resistance against oxidative stress (Brunet et al., 2004).

\section{DNA REPAIR}

As a result of ischemic stroke, oxidative stress will further cause damage to the DNA including oxidative base modifications and strand breaks. The accumulation of oxidative DNA lesions is attributed to the death of neurons. Therefore, the capacity for DNA repair plays an important role in the destiny of neurons in the condition of ischemic stress. Base excision repair (BER) is one of the most crucial parts in the DNA repair systems, which is the endogenous defense mechanism to rescue oxidative DNA damage (Srivastava et al., 1998).

$\mathrm{Ku} 70$ is one of the multifunctional DNA repair proteins, which triggers a DNA repair pathway by binding to broken DNA ends including double-strand breaks (Kim et al., 2001). With the decrease of Ku70 after focal cerebral ischemic injury, the repair process was found to be hindered (Kim et al., 2001). Sirt1 is found to regulate the acetylation of $\mathrm{Ku} 70$, thereby regulating the DNA repair pathways (Jeong et al., 2007).
Sirt1 also increases the activity of several other DNA repair pathways. The activation of poly [ADP-ribose] polymerase1 (PARP-1), a key mediator of cell death in excitotoxicity, ischemia, and oxidative stress, contributes to the depletion of $\mathrm{NAD}^{+}$and the release of apoptosis-inducing factor (AIF) from mitochondria, leading to cell death (Alano et al., 2010). The replenishment of cellular $\mathrm{NAD}^{+}$was demonstrated to confer marked neuroprotection effects by enhancing the DNA repair process against ischemic cell death (Wang et al., 2008). Kolthur-Seetharam et al. (2006) reported that SIRT1 modulates PARP-1 activity upon DNA damage. Sirt1 upregulation by resveratrol can reduce PARP-1 activity, however, there is a drastic increase in PAR synthesis leading to AIF-mediated cell death in the sirt1-null cells. This study indicated the protection mechanism of Sirt1 in ischemic stress. Further research is still needed to elucidate the full picture of the role of Sirt1 in DNA repair under ischemic stroke conditions.

\section{CEREBRAL BLOOD FLOW (CBF) MAINTAINING}

Sirt1 can deacetylate endothelial nitric oxide synthase (eNOS) and then maintain CBF (Hattori et al., 2014). In one particular study, mice overexpressing Sirtl were found to provide resistance to global ischemia by retaining cerebral perfusion up to $45 \%-$ $50 \%$ of baseline data (Hattori et al., 2015). Moreover, Hattori et al. (2014) found that endothelial Sirt1 deacetylates and activates eNOS, thus normalizing CBF. They further suggested that Sirt1-eNOS-NO system is responsible for the suppression of Sirt1-induced cerebral hypoperfusion. Sirt1 overexpression significantly attenuated blood-brain barrier (BBB) disruption, and an interaction of Sirt1 with eNOS facilitated NO-dependent vascular relaxation (Hattori et al., 2014). 


\section{FUNCTION ON ENERGY METABOLISM}

The brain consumes more energy per gram of tissue than any other organ (Taxin et al., 2014). The state of ischemia in the brain confers onto energy exhaustion. In turn, this metabolic stress aggravates the ischemic injury. Therefore, to promote cell survival and improve ischemic injury in the brain, it is important to find defense mechanisms in energy exhaustion (Guo et al., 2017). AMP-activated protein kinase (AMPK) is considered to be a major metabolic energy sensor. Decreased cellular energy charge (increased AMP/ATP ratio) activates AMPK and then regulates energy metabolic homeostasis (Briski et al., 2014; Zainal Abidin et al., 2015). Pharmacologic inhibition of AMPK was reported to alleviate ischemic damage in an ischemia model ( $\mathrm{Li}$ et al., 2007). Sirtl is suggested to be associated with the regulation of energy metabolism (Boutant and Canto, 2014). Sirt1-related AMPK pathway was reported to protect against ischemia stroke in some studies (Wang et al., 2011).

Estrogen was proved to have beneficial effects under cerebral ischemic stress through Sirt1-AMPK signaling pathway. Estrogen deficiency is considered as a risk factor for ischemic stroke in females after menopause (Ahnstedt et al., 2016), which partly explains the stroke-related gender differences (Persky et al., 2010; Sohrabji et al., 2013). Guo et al. (2017) showed that upregulating Sirt1 expression and then promoting AMPK activation can further regulate energy exhaustion, finally contributing to neuron survival under ischemic stress. In addition to estrogen, the adipokynin hormone, leptin, plays a role in severe energy depletion. Avraham et al. (2010) found that the expression of Sirtl gene increased in the cortex after leptin administration, which was in line with the reduction of infarct volume. This finding suggested that Sirt1 expression protects cortical neurons by modulating energetic status (Avraham et al., 2010).

Nicotinamide phosphoribosyltransferase (Nampt, also known as visfatin), the rate-limiting enzyme in mammalian $\mathrm{NAD}^{+}$ biosynthesis (Wang et al., 2012), plays several roles in protecting against ischemia, one of which was associated with the NamptSirt1-AMPK neuroprotective signaling pathway. Wang et al. (2012) reported that Nampt was significantly upregulated in the penumbra and infarct core of MCAO models. Inhibition and overexpression of Nampt augmented and reduced the infarction in MCAO rats, respectively. The upregulation of Nampt positively modulated $\mathrm{NAD}^{+}$levels and then upregulated Sirt1, contributing to LKB1 deacetylation, and thereby activating AMPK. This finding indicated that Nampt is an important protective factor in ischemic stroke (Wang et al., 2012).

Emerging studies showed that resveratrol could activate AMPK (Dasgupta and Milbrandt, 2007). Resveratrol was reported to activate a PDE-mediated signaling pathway that activates p-AMPK and Sirt1, thereby conferring cerebral ischemic tolerance (Wan et al., 2016).

The brain relies heavily on glucose for energy production (Koronowski et al., 2017). Production of ATP from glycolysis is crucial for fast axonal transport of vesicles (Zala et al., 2013), the energetic demand of action potential firing (Ashrafi et al., 2017), and the maintenance of synaptic ATP levels under energetic stress (Jang et al., 2016). Under ischemic conditions, it is important to utilize glucose more efficiently. Glycolysis can produce a significant amount of ATP to maintain ion gradients and delay depolarization. This effect is associated with neuronal Sirt1. One particular study demonstrated that resveratrol preconditioning increased glycolytic rate in a Sirt1dependent manner in neurons, thereby combating energetic stress in ischemic conditions (Koronowski et al., 2017).

\section{AUTOPHAGY}

Autophagic processes have been implicated as cell death mechanisms in the degradation and recycling of subcellular organelles (Kundu and Thompson, 2008). Generally, in the neuronal system, moderate autophagy is neuroprotective while inadequate or excess autophagy may lead to neuronal death (Shacka et al., 2008; Yue et al., 2008). Recently, autophagy has been recognized as a key process in ischemic stroke in addition to neurodegenerative diseases such as Alzheimer's and Parkinson's disease (Yue et al., 2008; Puyal et al., 2009). Sirt1 was first reported to regulate autophagy in Lee et al. (2008).

In addition to the Nampt-Sirt1-AMPK signaling pathway mentioned above, Nampt was demonstrated to have regulatory effects on autophagy under cerebral ischemic conditions (Wang et al., 2012). Wang et al. (2012) reported that Nampt promotes neuronal survival through inducing autophagy via regulating the TSC2-mTOR-S6K1 signaling pathway in a Sirt1-dependent manner during cerebral ischemia. Further studies are needed to find out more about the relationship between autophagy and ischemic stroke.

\section{IMPACT ON BLOOD-BRAIN BARRIER (BBB)}

Although most studies suggest that Sirt1 is a protective mediator in ischemic stroke, there are still a few studies that contradict these findings. Chen et al. (2018) reported that the activation of Sirt1 was associated with increased BBB permeability through AMPK-PGC1. Disruption of BBB and the cerebral edema that follows are the key pathogenic events contributing to neurological dysfunction and cerebral infarct after ischemic stroke (Chen et al., 2018). It is important to investigate further to find out the detailed mechanism and explain the contradiction between such studies.

\section{OTHER MECHANISMS}

Some studies reported that Sirt1 is important in the protection against ischemic stroke. Caloric restriction (CR) is defined as approximately $30 \%$ reduction in caloric intake, without compromising the maintenance of all essential nutrients (Redman et al., 2007). Short-term food restriction ( $40 \%$ less food over a 3-month period) was reported to attenuate ischemiainduced damage and improve functional recovery following global ischemia (Roberge et al., 2008). Proper period of CR can 
protect neurons from focal ischemic injury (Duan et al., 2001). Caloric restriction was found to increase the synthesis of Sirt1 and reduce the downregulation of Sirt1 expression in MCAO (Ran et al., 2015). Another study found that CDP-choline (citicoline), an intermediate in the biosynthesis of phosphatidylcholine, acted as Sirt1 activator by upregulating its expression and thereby reducing infarct volume in ischemic models (Hurtado et al., 2013). Further studies are still needed to elucidate the specific mechanisms.

\section{CONCLUSION AND PROSPECTS FOR FUTURE RESEARCH}

Since Sirt1 could exacerbate energy depletion and is associated with increased BBB permeability, there is still a controversy whether choosing Sirtl as a treatment target and increasing the Sirtl level would benefit the cerebral tissue under ischemic stress. Therefore, additional studies investigating the role of Sirt1 in ischemic stroke involving different cerebral cell types and animal

\section{REFERENCES}

Ahnstedt, H., McCullough, L. D., and Cipolla, M. J. (2016). The importance of considering sex differences in translational stroke research. Transl. Stroke Res. 7, 261-273. doi: 10.1007/s12975-016-0450-1

Alano, C. C., Garnier, P., Ying, W., Higashi, Y., Kauppinen, T. M., and Swanson, R. A. (2010). NAD+ depletion is necessary and sufficient for poly(ADP-ribose) polymerase-1-mediated neuronal death. J. Neurosci. 30, 2967-2978. doi: 10. 1523/jneurosci.5552-09.2010

Albers, G. W., Marks, M. P., Kemp, S., Christensen, S., Tsai, J. P., OrtegaGutierrez, S., et al. (2018). Thrombectomy for stroke at 6 to 16 hours with selection by perfusion imaging. N. Engl. J. Med. 378, 708-718. doi: 10.1056/ NEJMoa1713973

Ashrafi, G., Wu, Z., Farrell, R. J., and Ryan, T. A. (2017). GLUT4 mobilization supports energetic demands of active synapses. Neuron 93, 1-10. doi: 10.1016/j. neuron.2016.12.020

Avraham, Y., Davidi, N., Porat, M., Chernoguz, D., Magen, I., Vorobeiv, L., et al. (2010). Leptin reduces infarct size in association with enhanced expression of CB2, TRPV1, SIRT-1 and leptin receptor. Curr. Neurovasc. Res. 7, 136-143. doi: 10.2174/156720210791184943

Bordone, L., Motta, M. C., Picard, F., Robinson, A., Jhala, U. S., Apfeld, J., et al. (2006). Sirt1 regulates insulin secretion by repressing UCP2 in pancreatic beta cells. PLoS Biol. 4:e31. doi: 10.1371/journal.pbio.0040031

Boutant, M., and Canto, C. (2014). SIRT1 metabolic actions: integrating recent advances from mouse models. Mol. Metab. 3, 5-18. doi: 10.1016/j.molmet.2013. 10.006

Bramham, C. R., and Messaoudi, E. (2005). BDNF function in adult synaptic plasticity: the synaptic consolidation hypothesis. Prog. Neurobiol. 76, 99-125. doi: 10.1016/j.pneurobio.2005.06.003

Briski, K. P., Ibrahim, B. A., and Tamrakar, P. (2014). Energy metabolism and hindbrain AMPK: regulation by estradiol. Horm. Mol. Biol. Clin. Investig. 17, 129-136. doi: 10.1515/hmbci-2013-0067

Brunet, A., Sweeney, L. B., Sturgill, J. F., Chua, K. F., Greer, P. L., Lin, Y., et al. (2004). Stress-dependent regulation of FOXO transcription factors by the SIRT1 deacetylase. Science 303, 2011-2015. doi: 10.1126/science.10 94637

Canto, C., and Auwerx, J. (2009). PGC-1alpha, SIRT1 and AMPK, an energy sensing network that controls energy expenditure. Curr. Opin. Lipidol. 20, 98-105. doi: 10.1097/MOL.0b013e328328d0a4

Chan, P. H. (2004). Mitochondria and neuronal death/survival signaling pathways in cerebral ischemia. Neurochem. Res. 29, 1943-1949. doi: 10.1007/s11064-0046869-x models are necessary to arrive at more convincing conclusions. Besides, the relationship between Sirtl genetic polymorphism and ischemic stroke has not been researched extensively, which also needs further exploration. Altogether, Sirt1 is a promising therapeutic target for ischemic stroke for attenuating ischemic stress and improving stroke outcome.

\section{AUTHOR CONTRIBUTIONS}

All authors listed have made a substantial, direct and intellectual contribution to the work, and approved it for publication.

\section{FUNDING}

This work was partially supported by the grant of Clinical Research Innovation Plan of Shanghai General Hospital (Grant No. CTCCR-2018B03) and National Natural Science Foundation of China (Grant Nos. 81671251 and 81371410).

Chen, H. Z., Guo, S., Li, Z. Z., Lu, Y., Jiang, D. S., Zhang, R., et al. (2014). A critical role for interferon regulatory factor 9 in cerebral ischemic stroke. J. Neurosci. 34, 11897-11912. doi: 10.1523/JNEUROSCI.1545-14.2014

Chen, T., Dai, S. H., Li, X., Luo, P., Zhu, J., Wang, Y. H., et al. (2018). Sirt1-Sirt3 axis regulates human blood-brain barrier permeability in response to ischemia. Redox Biol. 14, 229-236. doi: 10.1016/j.redox.2017.09.016

Chen, W. Y., Wang, D. H., Yen, R. C., Luo, J., Gu, W., and Baylin, S. B. (2005). Tumor suppressor HIC1 directly regulates SIRT1 to modulate p53dependent DNA-damage responses. Cell 123, 437-448. doi: 10.1016/j.cell.2005. 08.011

Crack, P. J., and Taylor, J. M. (2005). Reactive oxygen species and the modulation of stroke. Free Radic. Biol. Med. 38, 1433-1444. doi: 10.1016/j.freeradbiomed. 2005.01.019

Cushman, M., Cantrell, R. A., McClure, L. A., Howard, G., Prineas, R. J., Moy, C. S., et al. (2008). Estimated 10-year stroke risk by region and race in the United States: geographic and racial differences in stroke risk. Ann. Neurol. 64, 507-513. doi: 10.1002/ana.21493

Dasgupta, B., and Milbrandt, J. (2007). Resveratrol stimulates AMP kinase activity in neurons. Proc. Natl. Acad. Sci. U.S.A. 104, 7217-7222. doi: 10.1073/pnas. 0610068104

Del Zoppo, G. J., Saver, J. L., Jauch, E. C., and Adams, H. P. Jr. (2009). Expansion of the time window for treatment of acute ischemic stroke with intravenous tissue plasminogen activator: a science advisory from the American Heart Association/American Stroke Association. Stroke 40, 2945-2948. doi: 10.1161/ strokeaha.109.192535

Della-Morte, D., Dave, K. R., DeFazio, R. A., Bao, Y. C., Raval, A. P., and PerezPinzon, M. A. (2009). Resveratrol pretreatment protects rat brain from cerebral ischemic damage via a sirtuin 1-uncoupling protein 2 pathway. Neuroscience 159, 993-1002. doi: 10.1016/j.neuroscience.2009.01.017

Donmez, G. (2012). The neurobiology of sirtuins and their role in neurodegeneration. Trends Pharmacol. Sci. 33, 494-501. doi: 10.1016/j. tips.2012.05.007

Donnan, G. A., Fisher, M., Macleod, M., and Davis, S. M. (2008). Stroke. Lancet 371, 1612-1623. doi: 10.1016/s0140-6736(08)60694-7

Doyle, K. P., Simon, R. P., and Stenzel-Poore, M. P. (2008). Mechanisms of ischemic brain damage. Neuropharmacology 55, 310-318. doi: 10.1016/j. neuropharm.2008.01.005

Duan, W., Lee, J., Guo, Z., and Mattson, M. P. (2001). Dietary restriction stimulates BDNF production in the brain and thereby protects neurons against excitotoxic injury. J. Mol. Neurosci. 16, 1-12. doi: 10.1385/JMN:16:1:1

Fu, B., Zhang, J., Zhang, X., Zhang, C., Li, Y., Zhang, Y., et al. (2014). Alphalipoic acid upregulates SIRT1-dependent PGC-1alpha expression and protects 
mouse brain against focal ischemia. Neuroscience 281, 251-257. doi: 10.1016/j. neuroscience.2014.09.058

Guo, J. M., Shu, H., Wang, L., Xu, J. J., Niu, X. C., and Zhang, L. (2017). SIRT1dependent AMPK pathway in the protection of estrogen against ischemic brain injury. CNS Neurosci. Ther. 23, 360-369. doi: 10.1111/cns.12686

Gutsaeva, D. R., Carraway, M. S., Suliman, H. B., Demchenko, I. T., Shitara, H., Yonekawa, H., et al. (2008). Transient hypoxia stimulates mitochondrial biogenesis in brain subcortex by a neuronal nitric oxide synthase-dependent mechanism. J. Neurosci. 28, 2015-2024. doi: 10.1523/jneurosci.5654-07.2008

Hattori, Y., Okamoto, Y., Maki, T., Yamamoto, Y., Oishi, N., Yamahara, K., et al. (2014). Silent information regulator 2 homolog 1 counters cerebral hypoperfusion injury by deacetylating endothelial nitric oxide synthase. Stroke 45, 3403-3411. doi: 10.1161/STROKEAHA.114.006265

Hattori, Y., Okamoto, Y., Nagatsuka, K., Takahashi, R., Kalaria, R. N., Kinoshita, M., et al. (2015). SIRT1 attenuates severe ischemic damage by preserving cerebral blood flow. Neuroreport 26, 113-117. doi: 10.1097/WNR. 0000000000000308

Hernandez-Jimenez, M., Hurtado, O., Cuartero, M. I., Ballesteros, I., Moraga, A., Pradillo, J. M., et al. (2013). Silent information regulator 1 protects the brain against cerebral ischemic damage. Stroke 44, 2333-2337. doi: 10.1161/ STROKEAHA.113.001715

Herskovits, A. Z., and Guarente, L. (2014). SIRT1 in neurodevelopment and brain senescence. Neuron 81, 471-483. doi: 10.1016/j.neuron.2014.01.028

Hurtado, O., Hernandez-Jimenez, M., Zarruk, J. G., Cuartero, M. I., Ballesteros, I., Camarero, G., et al. (2013). Citicoline (CDP-choline) increases Sirtuin1 expression concomitant to neuroprotection in experimental stroke. J. Neurochem. 126, 819-826. doi: 10.1111/jnc.12269

Jang, S., Nelson, J. C., Bend, E. G., Rodriguez-Laureano, L., Tueros, F. G., Cartagenova, L., et al. (2016). Glycolytic enzymes localize to synapses under energy stress to support synaptic function. Neuron 90, 278-291. doi: 10.1016/j. neuron.2016.03.011

Jeong, J., Juhn, K., Lee, H., Kim, S. H., Min, B. H., Lee, K. M., et al. (2007). SIRT1 promotes DNA repair activity and deacetylation of Ku70. Exp. Mol. Med. 39, 8-13. doi: 10.1038/emm.2007.2

Kelly, G. S. (2010). A review of the sirtuin system, its clinical implications, and the potential role of dietary activators like resveratrol: part 2. Altern. Med. Rev. 15, 313-328.

Kim, G. W., Noshita, N., Sugawara, T., and Chan, P. H. (2001). Early decrease in dna repair proteins, $\mathrm{Ku} 70$ and $\mathrm{Ku} 86$, and subsequent DNA fragmentation after transient focal cerebral ischemia in mice. Stroke 32, 1401-1407. doi: 10.1161/01. STR.32.6.1401

Kolthur-Seetharam, U., Dantzer, F., McBurney, M. W., de Murcia, G., and SassoneCorsi, P. (2006). Control of AIF-mediated cell death by the functional interplay of SIRT1 and PARP-1 in response to DNA damage. Cell Cycle 5, 873-877. doi: $10.4161 /$ cc.5.8.2690

Koronowski, K. B., Dave, K. R., Saul, I., Camarena, V., Thompson, J. W., Neumann, J. T., et al. (2015). Resveratrol preconditioning induces a novel extended window of ischemic tolerance in the mouse brain. Stroke 46, 2293-2298. doi: 10.1161/STROKEAHA.115.009876

Koronowski, K. B., Khoury, N., Saul, I., Loris, Z. B., Cohan, C. H., StradeckiCohan, H. M., et al. (2017). Neuronal SIRT1 (Silent information regulator 2 homologue 1) regulates glycolysis and mediates resveratrol-induced ischemic tolerance. Stroke 48, 3117-3125. doi: 10.1161/STROKEAHA.117.018562

Kou, D. Q., Jiang, Y. L., Qin, J. H., and Huang, Y. H. (2017). Magnolol attenuates the inflammation and apoptosis through the activation of SIRT1 in experimental stroke rats. Pharmacol. Rep. 69, 642-647. doi: 10.1016/j.pharep. 2016.12.012

Kundu, M., and Thompson, C. B. (2008). Autophagy: basic principles and relevance to disease. Annu. Rev. Pathol. 3, 427-455. doi: 10.1146/annurev. pathmechdis.2.010506.091842

Lee, I. H., Cao, L., Mostoslavsky, R., Lombard, D. B., Liu, J., Bruns, N. E., et al. (2008). A role for the NAD-dependent deacetylase Sirt1 in the regulation of autophagy. Proc. Natl. Acad. Sci. U.S.A. 105, 3374-3379. doi: 10.1073/pnas. 0712145105

Li, J., Zeng, Z., Viollet, B., Ronnett, G. V., and McCullough, L. D. (2007). Neuroprotective effects of adenosine monophosphate-activated protein kinase inhibition and gene deletion in stroke. Stroke 38, 2992-2999. doi: 10.1161/ STROKEAHA.107.490904
Li, L., Zhang, H. N., Chen, H. Z., Gao, P., Zhu, L. H., Li, H. L., et al. (2011). SIRT1 acts as a modulator of neointima formation following vascular injury in mice. Circ. Res. 108, 1180-1189. doi: 10.1161/circresaha.110.237875

Lv, H., Wang, L., Shen, J., Hao, S., Ming, A., Wang, X., et al. (2015). Salvianolic acid $B$ attenuates apoptosis and inflammation via SIRT1 activation in experimental stroke rats. Brain Res. Bull. 115, 30-36. doi: 10.1016/j.brainresbull.2015.05.002

Macrez, R., Ali, C., Toutirais, O., Le Mauff, B., Defer, G., Dirnagl, U., et al. (2011). Stroke and the immune system: from pathophysiology to new therapeutic strategies. Lancet Neurol. 10, 471-480. doi: 10.1016/S1474-4422(11)70066-7

Mattiasson, G., Shamloo, M., Gido, G., Mathi, K., Tomasevic, G., Yi, S., et al. (2003). Uncoupling protein-2 prevents neuronal death and diminishes brain dysfunction after stroke and brain trauma. Nat. Med. 9, 1062-1068. doi: 10. 1038/nm903

Mehta, S. L., Manhas, N., and Raghubir, R. (2007). Molecular targets in cerebral ischemia for developing novel therapeutics. Brain Res. Rev. 54, 34-66. doi: 10.1016/j.brainresrev.2006.11.003

Miao, Y., Zhao, S., Gao, Y., Wang, R., Wu, Q., Wu, H., et al. (2016). Curcumin pretreatment attenuates inflammation and mitochondrial dysfunction in experimental stroke: the possible role of Sirtl signaling. Brain Res. Bull. 121, 9-15. doi: 10.1016/j.brainresbull.2015.11.019

Moskowitz, M. A., Lo, E. H., and Iadecola, C. (2010). The science of stroke: mechanisms in search of treatments. Neuron 67, 181-198. doi: 10.1016/j. neuron.2010.07.002

Nada, S. E., Tulsulkar, J., Raghavan, A., Hensley, K., and Shah, Z. A. (2012). A derivative of the CRMP2 binding compound lanthionine ketimine provides neuroprotection in a mouse model of cerebral ischemia. Neurochem. Int. 61, 1357-1363. doi: 10.1016/j.neuint.2012.09.013

Nogueira, R. G., Jadhav, A. P., Haussen, D. C., Bonafe, A., Budzik, R. F., Bhuva, P., et al. (2018). Thrombectomy 6 to 24 hours after stroke with a mismatch between deficit and infarct. N. Engl. J. Med. 378, 11-21. doi: 10.1056/NEJMoa1706442

Pasinetti, G. M., Wang, J., Ho, L., Zhao, W., and Dubner, L. (2015). Roles of resveratrol and other grape-derived polyphenols in Alzheimer's disease prevention and treatment. Biochim. Biophys. Acta 1852, 1202-1208. doi: 10. 1016/j.bbadis.2014.10.006

Persky, R. W., Turtzo, L. C., and McCullough, L. D. (2010). Stroke in women: disparities and outcomes. Curr. Cardiol. Rep. 12, 6-13. doi: 10.1007/s11886009-0080-2

Petegnief, V., and Planas, A. M. (2013). SIRT1 regulation modulates stroke outcome. Transl. Stroke Res. 4, 663-671. doi: 10.1007/s12975-013-0277-y

Puyal, J., Vaslin, A., Mottier, V., and Clarke, P. G. (2009). Postischemic treatment of neonatal cerebral ischemia should target autophagy. Ann. Neurol. 66, 378-389. doi: 10.1002/ana.21714

Ran, M., Li, Z., Yang, L., Tong, L., Zhang, L., and Dong, H. (2015). Calorie restriction attenuates cerebral ischemic injury via increasing SIRT1 synthesis in the rat. Brain Res. 1610, 61-68. doi: 10.1016/j.brainres.2015.03.043

Redman, L. M., Heilbronn, L. K., Martin, C. K., Alfonso, A., Smith, S. R., and Ravussin, E. (2007). Effect of calorie restriction with or without exercise on body composition and fat distribution. J. Clin. Endocrinol. Metab. 92, 865-872. doi: 10.1210/jc.2006-2184

Roberge, M. C., Hotte-Bernard, J., Messier, C., and Plamondon, H. (2008). Food restriction attenuates ischemia-induced spatial learning and memory deficits despite extensive CA1 ischemic injury. Behav. Brain Res. 187, 123-132. doi: 10.1016/j.bbr.2007.09.002

Shacka, J. J., Roth, K. A., and Zhang, J. (2008). The autophagy-lysosomal degradation pathway: role in neurodegenerative disease and therapy. Front. Biosci. 13, 718-736. doi: 10.2741/2714

Shin, J. A., Lee, K. E., Kim, H. S., and Park, E. M. (2012). Acute resveratrol treatment modulates multiple signaling pathways in the ischemic brain. Neurochem. Res. 37, 2686-2696. doi: 10.1007/s11064-012-0858-2

Slemmer, J. E., Shacka, J. J., Sweeney, M. I., and Weber, J. T. (2008). Antioxidants and free radical scavengers for the treatment of stroke, traumatic brain injury and aging. Curr. Med. Chem. 15, 404-414. doi: 10.2174/0929867087834 97337

Sohrabji, F., Selvamani, A., and Balden, R. (2013). Revisiting the timing hypothesis: biomarkers that define the therapeutic window of estrogen for stroke. Horm. Behav. 63, 222-230. doi: 10.1016/j.yhbeh.2012.06.002

Srivastava, D. K., Berg, B. J., Prasad, R., Molina, J. T., Beard, W. A., Tomkinson, A. E., et al. (1998). Mammalian abasic site base excision repair. Identification 
of the reaction sequence and rate-determining steps. J. Biol. Chem. 273, $21203-$ 21209. doi: 10.1074/jbc.273.33.21203

St-Pierre, J., Drori, S., Uldry, M., Silvaggi, J. M., Rhee, J., Jager, S., et al. (2006). Suppression of reactive oxygen species and neurodegeneration by the PGC-1 transcriptional coactivators. Cell 127, 397-408. doi: 10.1016/j.cell.2006.09.024

Sun, A. Y., Wang, Q., Simonyi, A., and Sun, G. Y. (2010). Resveratrol as a therapeutic agent for neurodegenerative diseases. Mol. Neurobiol. 41, 375-383. doi: 10.1007/s12035-010-8111-y

Taxin, Z. H., Neymotin, S. A., Mohan, A., Lipton, P., and Lytton, W. W. (2014). Modeling molecular pathways of neuronal ischemia. Prog. Mol. Biol. Transl. Sci. 123, 249-275. doi: 10.1016/B978-0-12-397897-4.00014-0

Thomalla, G., Simonsen, C. Z., Boutitie, F., Andersen, G., Berthezene, Y., Cheng, B., et al. (2018). MRI-guided thrombolysis for stroke with unknown time of onset. N. Engl. J. Med. 379, 611-622. doi: 10.1056/NEJMoa1804355

Thompson, J. W., Narayanan, S. V., Koronowski, K. B., Morris-Blanco, K., Dave, K. R., and Perez-Pinzon, M. A. (2015). Signaling pathways leading to ischemic mitochondrial neuroprotection. J. Bioenerg. Biomembr. 47, 101-110. doi: 10. 1007/s10863-014-9574-8

Wan, D., Zhou, Y., Wang, K., Hou, Y., Hou, R., and Ye, X. (2016). Resveratrol provides neuroprotection by inhibiting phosphodiesterases and regulating the CAMP/AMPK/SIRT1 pathway after stroke in rats. Brain Res. Bull. 121, 255-262. doi: 10.1016/j.brainresbull.2016.02.011

Wang, P., Guan, Y. F., Du, H., Zhai, Q. W., Su, D. F., and Miao, C. Y. (2012). Induction of autophagy contributes to the neuroprotection of nicotinamide phosphoribosyltransferase in cerebral ischemia. Autophagy 8, 77-87. doi: 10 . 4161/auto.8.1.18274

Wang, P., Xu, T. Y., Guan, Y. F., Tian, W. W., Viollet, B., Rui, Y. C., et al. (2011). Nicotinamide phosphoribosyltransferase protects against ischemic stroke through SIRT1-dependent adenosine monophosphate-activated kinase pathway. Ann. Neurol. 69, 360-374. doi: 10.1002/ana.22236

Wang, R., Liu, Y. Y., Liu, X. Y., Jia, S. W., Zhao, J., Cui, D., et al. (2014). Resveratrol protects neurons and the myocardium by reducing oxidative stress and ameliorating mitochondria damage in a cerebral ischemia rat model. Cell Physiol. Biochem. 34, 854-864. doi: 10.1159/000366304

Wang, S., Xing, Z., Vosler, P. S., Yin, H., Li, W., Zhang, F., et al. (2008). Cellular NAD replenishment confers marked neuroprotection against ischemic cell death: role of enhanced DNA repair. Stroke 39, 2587-2595. doi: 10.1161/ STROKEAHA.107.509158
Wareski, P., Vaarmann, A., Choubey, V., Safiulina, D., Liiv, J., Kuum, M., et al. (2009). PGC-1\{alpha\} and PGC-1\{beta\} regulate mitochondrial density in neurons. J. Biol. Chem. 284, 21379-21385. doi: 10.1074/jbc.M109.018911

Xue, F., Huang, J. W., Ding, P. Y., Zang, H. G., Kou, Z. J., Li, T., et al. (2016). Nrf2/antioxidant defense pathway is involved in the neuroprotective effects of Sirtl against focal cerebral ischemia in rats after hyperbaric oxygen preconditioning. Behav. Brain Res. 309, 1-8. doi: 10.1016/j.bbr.2016.04.045

Yan, W., Fang, Z., Yang, Q., Dong, H., Lu, Y., Lei, C., et al. (2013). SirT1 mediates hyperbaric oxygen preconditioning-induced ischemic tolerance in rat brain. J. Cereb. Blood Flow Metab. 33, 396-406. doi: 10.1038/jcbfm.2012.179

Yue, Z., Wang, Q. J., and Komatsu, M. (2008). Neuronal autophagy: going the distance to the axon. Autophagy 4, 94-96. doi: 10.4161/auto.5202

Zainal Abidin, S., Tan, E. L., Chan, S. C., Jaafar, A., Lee, A. X., Abd Hamid, M. H., et al. (2015). DRD and GRIN2B polymorphisms and their association with the development of impulse control behaviour among Malaysian Parkinson's disease patients. BMC Neurol. 15:59. doi: 10.1186/s12883-015-0316-2

Zala, D., Hinckelmann, M. V., Yu, H., Lyra da Cunha, M. M., Liot, G., Cordelieres, F. P., et al. (2013). Vesicular glycolysis provides on-board energy for fast axonal transport. Cell 152, 479-491. doi: 10.1016/j.cell.2012.12.029

Zhang, S., Jiang, L., Che, F., Lu, Y., Xie, Z., and Wang, H. (2017). Arctigenin attenuates ischemic stroke via SIRT1-dependent inhibition of NLRP3 inflammasome. Biochem. Biophys. Res. Commun. 493, 821-826. doi: 10.1016/j.bbrc.2017.08.062

Zhu, H. R., Wang, Z. Y., Zhu, X. L., Wu, X. X., Li, E. G., and Xu, Y. (2010). Icariin protects against brain injury by enhancing SIRT1-dependent PGC1alpha expression in experimental stroke. Neuropharmacology 59, 70-76. doi: 10.1016/j.neuropharm.2010.03.017

Conflict of Interest Statement: The authors declare that the research was conducted in the absence of any commercial or financial relationships that could be construed as a potential conflict of interest.

Copyright (c) 2018 Zhang, Zhang and Wu. This is an open-access article distributed under the terms of the Creative Commons Attribution License (CC BY). The use, distribution or reproduction in other forums is permitted, provided the original author(s) and the copyright owner(s) are credited and that the original publication in this journal is cited, in accordance with accepted academic practice. No use, distribution or reproduction is permitted which does not comply with these terms. 